\title{
APOYOS Y AUTODETERMINACIÓN EN LA ESCUELA INCLUSIVA. FACTORES RELACIONADOS CON LA OBTENCIÓN DE RESULTADOS PERSONALES POSITIVOS
}

\section{SUPPORTS AND SELF-DETERMINATION IN THE INCLUSIVE SCHOOL. FACTORS RELATED TO OBTANING PERSONAL POSITIVE RESULTS}

\author{
Jesús Miguel Muñoz-Cantero ${ }^{1}$ \\ Luisa Losada-Puente \\ Eva María Espiñeira-Bellón \\ Universidad de A Coruña, España.
}

\section{RESUMEN}

Las propuestas educativas actuales se fundamentan en el principio de atención a la diversidad, desde el que se pretende dotar al alumnado de los apoyos y medidas necesarias para alcanzar una plena autodeterminación en la escuela inclusiva. El objetivo del presente estudio es evaluar el nivel de autodeterminación del alumnado adolescente con y sin Necesidades Específicas de Apoyo Educativo (NEAE) y la influencia de las medidas de apoyo sobre la consecución de un mayor nivel de autodeterminación, con el fin de proporcionar una serie de pautas de intervención en las aulas que orienten el aprendizaje y expresión de comportamientos autodeterminados. Se emplea una metodología cuantitativa, de tipo descriptivo-exploratorio, para la evaluación de la autodeterminación en cuatro dimensiones: autonomía, autorregulación, empoderamiento y autoconocimiento. La muestra se compone de 1,746 estudiantes, y el instrumento utilizado es la Escala ARC-INICO (Verdugo et al., 2015b). Los resultados muestran niveles adecuados de autodeterminación, siendo significativamente inferiores en el alumnado con

1 Correspondencia: Jesús Miguel Muñoz-Cantero. Dpto. Didácticas Específicas y Métodos de Investigación y Diagnóstico en Educación. Facultad de Ciencias de la Educación. Universidade da Coruña. Campus de Elviña, s/n, C.P. 15071. A Coruña. Correo-e: jesus.miguel.munoz@udc.es, web: http://giace.udc.es/ 
NEAE, sobre todo en aquel que recibe apoyo de carácter intensivo (Adaptación Curricular Individualizada, Programas de Diversificación Curricular, combinación entre agrupamiento flexible y refuerzo educativo). Estos resultados invitan a reflexionar sobre el tipo y la calidad de los recursos de los centros y sobre la actuación del profesorado para atender a la diversidad del alumnado y la promoción de su autodeterminación desde la perspectiva de la calidad de vida. Se proponen pautas de orientación e intervención para la instrucción en autodeterminación en el aula y desde los departamentos de orientación.

Palabras clave: autodeterminación, atención a la diversidad, necesidades educacionales, apoyo educativo, orientación educativa.

\section{ABSTRACT}

Current educational proposals are based on the principle of attention to diversity. It expects to give all students the supports and resources needed to achieve a full self-determination in the inclusive school. The aim of the present study is to assess the level of self-determination of students with and without Special Educational Needs (SEN), as well as the influence of the educational supports on achieving a higher level of self-determination, in order to provide a set of guidelines for intervention in classrooms that routed the learning and expression of self-determined behaviors in young students. A quantitative methodology, descriptive-exploratory, is used to assess self-determination in four domains: autonomy, self-regulation, empowerment and selfknowledge. The ARC-INICO scale (Verdugo et al., 2015b) is applied to a sample composed by 1,746 students. The results show adequate level of self-determination. It is significantly lower in students with SEN, especially those who receives intensive support (Individualized Educational Programs, Curricular Diversification Programs, combination of flexible grouping and educational support). These findings invite reflection on the type and quality of the resources of schools and the teachers' actions to address student diversity and the promotion of self-determination from the perspective of quality of life. Guidelines for counseling and intervention for instruct selfdetermination in the classroom and in the guidance departments are proposed.

Key Words: self-determination, attention to diversity, special needs students, academic support services, educative guidance.

\section{Introducción}

La educación en España ha experimentado transformaciones encaminadas al desarrollo de escuelas inclusivas, en las que se prioriza el respeto a las individualidades y la respuesta a las necesidades de cada alumno y alumna partiendo no de sus limitaciones, sino de sus potencialidades y capacidades, apoyándolos en su transición a la vida adulta y analizando el modo en que se producen los procesos de exclusión y devaluación educativa y social. Esto es, ofrecer una verdadera atención a la diversidad del alumnado en las aulas (Arnáiz, 2009; Luque y Luque, 2015). Dichas actuaciones se materializan, principalmente, en la dotación de medidas de apoyo para alumnado que presenta Necesidades Específicas de Apoyo Educativo (NEAE), es decir, para aquellos que requieran una atención educativa diferente a la ordinaria, por presentar necesidades educativas especiales, por dificultades específicas de aprendizaje, TDAH, por sus altas capacidades intelectuales, por haberse incorporado tarde al sistema educativo, o por condiciones personales o de historia escolar, puedan alcanzar el máximo desarrollo posible de sus capacidades personales y, en todo caso, los objetivos establecidos con carácter general para todo 
el alumnado (Ley Orgánica 8/2013, de 9 de diciembre, de Mejora de la Calidad Educativa, LOMCE, art. 71, apart. 2).

La LOMCE (2013), continuando con la propuesta de la Ley Orgánica 2/2006, de 3 de mayo, de Educación (LOE), alude a diversas tipologías de NEAE: por un lado, las Necesidades Educativas Especiales (NEE) -presentes en el alumnado con Discapacidad Intelectual y Del Desarrollo (DI/DD) -, y por el otro, al alumnado con Dificultades Significativas de Aprendizaje (DSA), alumnado con Retraso Escolar (RE) y alumnado con Altas Capacidades. Se pretende ofrecer medidas (ordinarias y/o extraordinarias) adecuadas y ajustadas a las necesidades individuales. Dichas medidas abarcan un continuo de respuestas graduadas con diferentes niveles de concreción curricular, desde aquellas que se consideran medidas ordinarias o de carácter general (las más comunes: refuerzo educativo, agrupamiento flexible) hasta las medidas extraordinarias de atención a la diversidad, siendo las más utilizadas la Adaptación Curricular Individualizada $(\mathrm{ACl})$ con diferentes niveles de intensidad y los Programas de Diversificación Curricular (PDC) para la etapa de $3^{\circ}$ y $4^{\circ}$ de ESO.

Este marco legislativo recupera el clásico concepto de alumnado con discapacidad empleado tradicionalmente en la Educación Especial, que predispone a clasificar al alumnado según sus características y anula el carácter normalizador e inclusivo de las escuelas, pues restringe el concepto de NEE a su equiparación con el alumnado con discapacidad o trastornos del comportamiento, para el cual se diseñan medidas homogeneizadoras, que acrecientan la desigualdad en las aulas y contradicen el principio de atención a la diversidad (Domínguez, López y Vázquez, 2016; González Gil, 2011). A ellos se suman otros dos condicionantes:

El primero es el déficit en la respuesta educativa ofrecida al alumnado con NEAE en la etapa de la Educación Secundaria Obligatoria (ESO; Arnáiz, 2009; Rodríguez, 2013) que, en la Comunidad Autónoma de Galicia se subdivide, principalmente, en medidas de refuerzo educativo y apoyo del profesorado, como medidas ordinarias, y los apoyos fuera del aula (Pedagogía Terapéutica y Audición y Lenguaje), Adaptaciones Curriculares y Programas de Diversificación Curricular, como medidas extraordinarias (Domínguez et al., 2016).

El segundo se refiere a la pervivencia de ciertas formas de pensamiento social ancladas en la visión del alumnado con necesidad de apoyo como incapaz, vulnerable, dependiente,... provocando que las escuelas pongan en duda la capacidad de este alumnado para ser autodeterminado y asuman que es una característica que se tiene o no se tiene (Beadle-Brown et al., 2015; Gómez-Vela, Verdugo, González-Gil, Badía y Wehmeyer, 2012; Wehmeyer et al., 2011).

La necesidad de apoyo no supone un freno a la autodeterminación, sino un medio fundamental para su consecución, pues contribuyen a la mejora del funcionamiento humano al reducir los desajustes entre competencias personales y demandas ambientales (Schalock et al., 2011). Con los apoyos adecuados, cualquier individuo podrá adquirir las habilidades y capacidades que les permitan tomar el control, decidir y responsabilizarse de sus acciones, entrando en juego dos elementos fundamentales: características individuales y entorno. Este último es clave en las primeras etapas de desarrollo (infancia y adolescencia), pues a través de la instrucción y los apoyos familiares, escolares y comunitarios, así como de las oportunidades y estímulos que reciba el individuo, logrará convertirse en una persona autodeterminada (Pallisera et al., 2014; Wehmeyer y Abery, 2013).

Educar a la adolescencia en autodeterminación supone "un profundo cambio en la manera de concebir y planificar la provisión de apoyos en el contexto escolar, social y familiar, prestando atención a los roles a desempeñar por los distintos agentes en cada uno de estos contextos" (Peralta y Arellano, 2014, p. 67). Los apoyos escolares deben configurarse como una red de relaciones, coordinación e interdependencia entre profesorado, familias y comunidad, quienes 
deben aunar sus esfuerzos para favorecer la promoción de la autodeterminación de la adolescencia con NEAE, con NEE y también del resto del alumnado.

Diversos estudios demuestran la eficacia de los apoyos en la adquisición y expresión de comportamientos autodeterminados en la etapa adolescente, sobre todo en jóvenes con NEE, sobre la obtención de resultados personales más positivos durante la etapa escolar: mayor éxito académico e implicación en la toma de decisiones educativas (Erickson, Noonan, Zheng y Brussow, 2015; Marfull-Jensen, Flanagan y Ossa, 2013; Shogren, Wehmeyer, Palmer y Paek, 2013; Shogren et al., 2015; Wehmeyer, Field y Thoma, 2012) y al finalizar la escolarización: mayor bienestar, autorregulación, autonomía e independencia, consecución de empleo y, en definitiva, mejor calidad de vida (Marfull-Jensen et al., 2013; Shogren y Shaw, 2016; Shogren, Wehmeyer, Palmer, Rifenbank y Little, 2015; Wehmeyer et al., 2012). Por el contrario, se ha evidenciado que la falta de oportunidades y experiencias en la etapa escolar, sobre todo en el alumnado con necesidad de apoyos intermitentes o intensivos, da lugar a peores resultados al finalizar la etapa educativa (abandono escolar, falta de apoyo y de conexión con un empleo significativo, empleo remunerado en ambientes segregados, ...; Shogren et al., 2015).

Teniendo en cuenta esto, este trabajo parte del reconocimiento de que las limitaciones en el funcionamiento intelectual y la intensidad de apoyo requerido por el alumnado no suponen un impedimento para la toma de decisiones y la proyección de la propia vida hacia la adultez. Así mismo, la postura que se adopta es la de considerar que todo el alumnado puede llegar a ser sujeto de algún apoyo en mayor o menor medida y, por tanto, su nivel de autodeterminación puede verse condicionado por la provisión de un adecuado entrenamiento y capacitación. Pero también se reconoce la existencia de alumnado cuya necesidad de apoyo puede ser más evidente, por lo que el estudio de sus necesidades y de sus demandas puede ofrecer una visión más clara de su situación y facilitar la propuesta de pautas educativas que orienten el trabajo en las aulas hacia la consecución de niveles superiores de autocontrol, autonomía, empoderamiento y autoconocimiento.

El objetivo central de este estudio es proporcionar orientaciones y pautas de intervención en las aulas que encaminen el aprendizaje y expresión de comportamientos autodeterminados en el alumnado adolescente, a partir de la evaluación de sus niveles de autodeterminación y el análisis de la influencia de las medidas de apoyo sobre su consecución. Para ello, se da respuesta a los siguientes objetivos específicos:

- Evaluar el nivel de autodeterminación del alumnado adolescente con y sin NEAE, a partir de las puntuaciones obtenidas en cada característica del constructo (autonomía, autorregulación, empoderamiento y autoconocimiento), en base al Modelo Funcional de Autodeterminación de Wehmeyer (2003).

- Analizar la presencia o no de diferencias estadísticamente significativas entre alumnado con y sin NEAE en términos de autodeterminación, autonomía, autorregulación, empoderamiento y autoconocimiento.

- Contrastar los niveles de autodeterminación del alumnado que presentan NEAE en función del tipo de apoyos recibidos (ausencia de apoyos, Adaptación Curricular Individualizada $(\mathrm{ACl})$ o Programas de Diversificación Curricular (PDC), Agrupamiento Flexible (AF) o Refuerzo Educativo (RE).

- Proponer una serie de orientaciones para la evaluación y diseño de intervenciones en el aula, así como algunas actividades específicas al alcance del profesorado y del equipo de orientación de los centros educativos. 


\section{Método}

\section{Muestra}

El estudio se ha realizado con una muestra no probabilística discrecional de 1,746 estudiantes entre 12-18 años que cursan Educación Secundaria Obligatoria (ESO) en la Comunidad Autónoma de Galicia, formada por un total de 88,655 adolescentes en el curso 2014/2015 (Instituto Galego de Estadística, IGE, 2015). Dado que se trata de una población finita y conocida, en el cálculo del tamaño muestral se propuso un tamaño mínimo de $\mathrm{N}=1,054$ estudiantes, con un nivel de confianza de $95 \%$ y un margen de error de 3\%, utilizando para el cálculo del tamaño muestral las recomendaciones de Hernández Pina (1995) y Manzano y Braña (2003). La media de edad es $13.7($ D.T.= 1.4), de los cuales 942 son hombres $(54 \%)$ y 804 mujeres $(46 \%)$. La muestra integra 563 estudiantes con NEAE (32.2\%) de los cuales 228 alumnos/as (40.3\%) presentan Discapacidad Intelectual y Del Desarrollo (DI/DD), 152 alumnos/as con Dificultades Significativas de Aprendizaje (DSA, 26.9\%) y 169 alumnos/as con Retraso Escolar (29.9\%). La información referente a las NEAE del alumnado y el tipo de apoyo que reciben fue facilitada por el equipo de orientación de los centros. La tabla 1 presenta las medidas de apoyo ofrecidas al alumnado con NEAE en general y en función del tipo de NEAE.

TABLA 1. Tabla de contingencia NEAE * Medidas Específicas de Apoyo Educativo

\begin{tabular}{|c|c|c|c|c|c|c|c|}
\hline & & \multicolumn{5}{|c|}{ Medidas educativas específicas } & \multirow[b]{2}{*}{ Tota } \\
\hline & & Ninguna & $\mathrm{ACl} / \mathrm{PDC}$ & Ref. Ed. & $\mathrm{AF}$ & Ref. Ed. y AF & \\
\hline \multirow{2}{*}{ DI/DD } & $\mathrm{N}$ & 27 & 73 & 66 & 24 & 37 & 563 \\
\hline & $\%$ & 11.9 & 32.2 & 29.1 & 10.5 & 16.3 & 100 \\
\hline \multirow{2}{*}{ DSA } & $\mathrm{N}$ & 19 & 35 & 78 & 18 & 1 & 563 \\
\hline & $\%$ & 12.5 & 23.2 & 51.7 & 11.9 & 0.7 & 100 \\
\hline \multirow{2}{*}{ RE } & $\mathrm{N}$ & 16 & 6 & 102 & 34 & 9 & 563 \\
\hline & $\%$ & 9.5 & 3.6 & 61.1 & 20.4 & 5.4 & 100 \\
\hline Total & $\mathrm{N}$ & 80 & 114 & 246 & 76 & 47 & 563 \\
\hline
\end{tabular}

Nota. Siglas y abreviaturas: NEAE - Necesidades Específicas de Apoyo Educativo; DI/DD - Discapacidad Intelectual y otras Discapacidades del Desarrollo; DSA - Dificultades Significativas de Aprendizaje; RE - Retraso Escolar; ACI/PDC - Adaptación Curricular Individualizada/ Programa de Diversificación Curricular; Ref.Ed. - Refuerzo Educativo; AF - Agrupamiento Flexible.

En la tabla 1 se evidencia un predominio de la medida de refuerzo educativo (43.7\%) fuera del aula y sobre todo, para el alumnado con Retraso Escolar (61.1\%) que deriva de dificultades socioeconómicas, familiares, integración tardía al Sistema Educativo, desconocimiento del idioma español por procedencia extranjera, etc. Destacan también las medidas de Adaptación Curricular Individualizada $(\mathrm{ACl})$ para alumnado de $1^{\circ}$ y $2^{\circ}$ de ESO y los Programas de Diversificación Curricular (PDC) para alumnado de $3^{\circ}$ y $4^{\circ}$ de ESO, siendo destacable en alumnado con discapacidad (32.2\%) y con Dificultades Significativas de Aprendizaje (23.2\%). La combinación de agrupamiento flexible (para Lenguas o Matemáticas, en aulas específicas) y refuerzo educativo es una medida bastante empleada en los centros educativos como sustituta de la ACl o del PDC en alumnado con Discapacidad Intelectual y Del Desarrollo (16.3\%).

\section{Instrumentos}

Se ha empleado la Escala ARC-INICO de Evaluación de la Autodeterminación (Verdugo Alonso et al., 2015b), compuesta por 61 ítems organizados en cuatro secciones: autonomía (25 
ítems), autorregulación (12 ítems), empoderamiento (14 ítems) y autoconocimiento (10 ítems). La escala se fundamenta en el Modelo Funcional de Autodeterminación de Wehmeyer (2003) y su estructura resulta de la adaptación del cuestionario The ARC' Self Determination Scale (Wehmeyer, 1995; Wehmeyer y Kelchner, 1995). El uso de la ARC-INICO en España aún no es muy extendido, dada su reciente publicación, pero presenta adecuadas propiedades psicométricas en su aplicación inicial con adolescentes de 12-19 años con Discapacidad Intelectual y Del Desarrollo y con Dificultades Significativas de Aprendizaje (total $\alpha=.92$; rango $\alpha=.90-.81$ dimensiones), y una estructura en cuatro factores independientes, confirmada mediante un Análisis de los Componentes Principales (AFC) y que, en conjunto, permiten evaluar cada una de las características de la autodeterminación (Verdugo et al., 2014). Complementariamente, Vicente, Verdugo, Gómez, Fernández y Guillén (2015) analizaron las propiedades psicométricas de la escala en su aplicación a 99 estudiantes con discapacidad intelectual (11-19 años), aportando datos adicionales sobre su fiabilidad (total $\alpha=.93$; rango $\alpha=.93-.71$ dimensiones) y Verdugo et al. (2015a) comprobaron, mediante Análisis Factorial Confirmatorio (ACF) la validez de la estructura interna de la ARC-INICO, confirmando su correcto ajuste a la estructura jerárquica propuesta.

TABLA 2. Distribución de los resultados y coeficiente de consistencia interna de los componentes de la ARC-INICO

\begin{tabular}{lcccccc}
\hline Componentes & $N^{0}$ ítems & Media & $D T$ & $\begin{array}{c}\alpha \text { presente } \\
\text { estudio }\end{array}$ & $\begin{array}{c}\alpha \text { (Verdugo et al., } \\
\text { 2014) }\end{array}$ & $\begin{array}{c}\alpha \text { (Vicente et al., } \\
\text { 2015) }\end{array}$ \\
\hline Autonomía & 25 & 2.2 & .36 & .92 & .90 & .92 \\
Autorregulación & 12 & 2.85 & .53 & .94 & .84 & .69 \\
Empoderamiento & 14 & 3.09 & .46 & .89 & .85 & .78 \\
Autoconocimiento & 10 & 3.24 & .42 & .81 & .81 & .71 \\
\hline Total & 61 & 2.71 & .34 & .95 & .92 & .93 \\
\hline
\end{tabular}

La tabla 2 presenta los valores de la media, la desviación típica y el $\alpha$ Cronbach evaluando la consistencia de los ítems por dimensión. Evidenciamos en nuestro estudio una consistencia interna superior a la de su validación inicial en tres de los cuatro componentes de la autodeterminación y para el total de la escala.

TABLA 3. Varianza total explicada en cuatro factores

\begin{tabular}{cccc}
\hline Componentes & $N^{0}$ ítems & \% varianza & \% varianza acumulada \\
\hline 1 & 22 & 28.33 & 28.33 \\
2 & 21 & 7.05 & 35.38 \\
3 & 12 & 6.23 & 41.61 \\
4 & 6 & 5.66 & 47.27 \\
\hline
\end{tabular}

La estructura factorial difiere ligeramente de la propuesta por Verdugo et al. (2014) quienes detectaron que el primer factor explicaba el $40 \%$ de la varianza total (no proporcionan datos adicionales de la proporción de varianza explicada por los cuatro factores). Respecto al estudio de Vicente et al. (2015), realizaron un Análisis Factorial Exploratorio (AFE), forzando la estructura en cuatro factores, con los que explican un $36.72 \%$ de la varianza.

Respecto a la estructura factorial obtenida en este estudio, el componente 1 integra la mayoría de las habilidades relacionadas con la autonomía, excepto aquellas referidas a los cuidados básicos y varios ítems que los autores encuadran en la sección empoderamiento 
(cuando tengo que elegir, suelo hacerlo bien; cuando sea necesario seré capaz de tomar decisiones importantes para mí; tomo mis propias decisiones) pero cuyo contenido se relaciona íntimamente con la autonomía. El componente 2 integra habilidades de empoderamiento y autoconocimiento, dos aspectos muy relacionados. El componente 3 se mantiene fiel al instrumento original integrando todos los ítems relacionados con la autorregulación. Por último, el componente 4 incluye ítems encuadrados originalmente en la dimensión de autonomía y cuyo contenido refiere a las habilidades básicas de la vida diaria (preparar alimentos, hacer tareas del hogar, ordenar las cosas, cuidado de la ropa, cuidado de la imagen e higiene personal y cuidados básicos ante algún daño).

\section{Procedimiento}

Seleccionado el instrumento de recogida de información y la población objeto de estudio, se contactó con los centros educativos gallegos por correo electrónico y telefónicamente para explicar el estudio y obtener consentimiento mediante un documento escrito dirigido al equipo directivo y a las familias para aplicar el cuestionario al alumnado. La ARC-INICO se aplicó durante el curso 2014/2015 en las cuatro provincias gallegas (A Coruña, Lugo, Orense y Pontevedra). Las aulas participantes debían cumplir el requisito de contar, por lo menos, con un alumno o alumna con NEAE, así como que todo el alumnado tuviese habilidades de lectoescritura y comprensión lectora. En algunos casos, fue requerido apoyo del profesorado e investigadores para cumplimentar la escala por parte del alumnado con necesidades de apoyo más extensivas, basándose en la lectura en voz alta, transcripción de respuestas y explicación de los ítems de difícil comprensión.

\section{Análisis de datos}

Los datos fueron codificados e introducidos en la matriz de datos del programa estadístico IBM SPSS Statistics 23. Tras comprobar la consistencia interna (tabla 2) y la estructura del instrumento (tabla 3), se agruparon los ítems en las cuatro secciones que componen al cuestionario: autonomía, autorregulación, empoderamiento, y autoconocimiento. Se calcularon las puntuaciones totales mediante la suma de los ítems en cada sección y se transformaron a una escala 0-100, para hacer las comparaciones de forma adecuada (Gómez-Vela et al., 2012).

Con el fin de responder a los objetivos de: (a) evaluar del nivel de autodeterminación del alumnado con y sin NEAE, (b) analizar la presencia de diferencias estadísticamente significativas entre ambos grupos en el nivel de autodeterminación y sus dimensiones, y (c) contrastar los niveles de autodeterminación en función de las medidas de apoyo recibidas por el alumnado con $\mathrm{NEAE}$, se realizaron dos análisis que responden a los varios objetivos del estudio:

- Análisis descriptivo de las puntuaciones obtenidas por el alumnado con y sin NEAE en autodeterminación y sus dimensiones.

- Comprobación de la existencia de diferencias estadísticamente significativas en las puntuaciones del alumnado en función de la presencia o no de NEAE y el tipo y según las medidas específicas de apoyo educativo que se ofrecen al alumnado con NEAE, a fin de detectar medidas necesarias de intervención en áreas específicas. 


\section{Resultados}

Inicialmente, se comprobó la normalidad de la distribución mediante la prueba de bondad de ajuste de Kolmogorov-Smirnov (K-S= 3.198; $p<.005)$ y la homogeneidad de la varianza a través del estadístico de Levene $(\mathrm{W}=3.921 ; \mathrm{p}<.005)$ que indican el incumplimiento de los supuestos de normalidad, por lo que no es posible realizar contrastes paramétricos de los datos, empleando en su lugar estadística no paramétrica.

TABLA 4. Estadísticos descriptivos de las puntuaciones de la ARC-INICO

\begin{tabular}{|c|c|c|c|c|c|c|c|c|c|}
\hline & \multicolumn{3}{|c|}{ Alumnado en general } & \multicolumn{3}{|c|}{ Alumnado C/NEAE } & \multicolumn{3}{|c|}{ Alumnado S/NEAE } \\
\hline Componentes & $P T$ & $P E$ & $P_{50}$ & $P T$ & $P E$ & $P_{50}$ & $P T$ & $P E$ & $P_{50}$ \\
\hline Autodeterminación & 165.04 & 75.4 & 76.7 & 147.6 & 67.4 & 67.1 & 173.4 & 79.2 & 79.5 \\
\hline Autonomía & 55.2 & 73.6 & 74.6 & 47.7 & 63.6 & 62.7 & 58.8 & 78.4 & 78.7 \\
\hline Autorregulación & 34.1 & 71.1 & 75 & 29.8 & 62.1 & 62.5 & 36.2 & 75.4 & 75 \\
\hline Empoderamiento & 43.2 & 77.2 & 76.8 & 39.5 & 70.5 & 71.4 & 45.04 & 80.4 & 80.4 \\
\hline Autoconocimiento & 32.4 & 81.1 & 76.7 & 30.6 & 76.6 & 77.5 & 33.3 & 83.2 & 82.5 \\
\hline
\end{tabular}

Nota. Siglas P.T.: Puntuación Total; P.E.: Puntuación Escala (0-100); C/NEAE: Con Necesidades Educativas Especiales; S/NEAE: Sin Necesidades Educativas Especiales.

\section{Análisis descriptivo de las puntuaciones de la ARC-INICO}

La tabla 4 presenta las puntuaciones obtenidas en referencia al nivel de autodeterminación del alumnado, siendo la puntuación máxima posible 219, y a las características que componen al constructo: autonomía (máx. 75 puntos), autorregulación (máx. 48 puntos), empoderamiento (máx. 56 puntos) y autoconocimiento (máx. 40 puntos). Se incluyen las puntuaciones transformadas en una escala 0-100 y el $P_{50}$ o Mediana.

Se observa un nivel adecuado de autodeterminación (P.T.=165.04; P.E.=75.4), situándose el $50 \%$ del total de las puntuaciones por encima del $P_{50}=70$. Igualmente, los componentes de la autodeterminación obtienen valores superiores al 70\% en todos los casos, superando el $70 \%$ en todos los casos.

Se aprecian diferencias entre alumnado con NEAE y sin ellas. Descriptivamente, se puede señalar que el alumnado con NEAE experimenta un nivel inferior de autodeterminación (más de 10 puntos por debajo del alumnado sin NEAE) y menores habilidades y oportunidades para actuar de un modo autónomo (14.8 puntos por debajo), autorregulado (13.3 puntos por debajo), así como un peor empoderamiento (9.9 puntos por debajo) y autoconocimiento (6.6 puntos por debajo) en comparación con sus iguales sin NEAE.

Estos resultados deben ser entendidos, no en términos negativos, sino como una información valiosa para detectar las áreas en las que sería más adecuado poner atención para la orientación e intervención tanto con el alumnado con NEAE como sin ellas. 
Análisis de las diferencias entre alumnado con y sin NEAE en función del tipo y necesidad de apoyo

A continuación, analizamos las diferencias entre alumnado con y sin NEAE en los niveles de autodeterminación y sus componentes, según el tipo de apoyos recibidos (ausencia de apoyos, refuerzo educativo, agrupamiento flexible, diversificación curricular, $\mathrm{ACl}$ ). La prueba $\mathrm{H}$ de KruskalWallis, evidencia diferencias significativas en todas las variables contrastadas: autodeterminación $\left(\chi^{2}=850.868 ; p=.000\right)$, autonomía $\left(\chi^{2}=815.014 ; p=.000\right)$, autorregulación $\left(\chi^{2}=596.651 ; p=\right.$ $.000)$, empoderamiento $\left(\chi^{2}=478.849 ; p=.000\right)$ y autoconocimiento $\left(\chi^{2}=244.713 ; p=.000\right)$.

Empleamos como variable de agrupación la presencia o no de NEAE diferenciando:

- Ausencia de NEAE.

- Discapacidad Intelectual y Del Desarrollo (DI/DD).

- Dificultades Significativas de Aprendizaje (DSA).

- Retraso Escolar (RE).

Para conocer dónde residen dichas diferencias empleamos la prueba $U$ de Mann-Whitney (tabla 5), obteniendo diferencias significativas entre todos los grupos contrastados. Hallamos el tamaño del efecto (effect size) $d$ de Cohen (1988), que determina la fuerza o magnitud de la significación estadística para pruebas no paramétricas, donde $Z$ es sensible al tamaño de la muestra, y cuya interpretación es: $\mathrm{d} \leq .1$ efecto pequeño, $\mathrm{d}$ entre .1 y .3 efecto de magnitud media $y \mathrm{~d} \geq .5$ efecto de alta magnitud.

TABLA 5. Comparación de los niveles de autodeterminación en función del tipo de NEAE

\begin{tabular}{|c|c|c|c|c|c|}
\hline \multicolumn{6}{|c|}{ Autodeterminación } \\
\hline & $N$ & Rango promedio & $Z$ & $d$ & $P$ \\
\hline S/NEAE & 1516 & 1054.06 & -25.61 & -.66 & .000 \\
\hline DI/DD & 307 & 210.47 & & & \\
\hline S/NEAE & 1516 & 905.38 & -15.59 & -.40 & .000 \\
\hline DSA & 170 & 291.63 & & & \\
\hline S/NEAE & 1516 & 886.01 & -7.84 & -.20 & .000 \\
\hline RE & 189 & 588.24 & & & \\
\hline DI/DD & 307 & 196.14 & -9.13 & -.23 & .000 \\
\hline DSA & 170 & 316.4 & & & \\
\hline DI/DD & 309 & 175.8 & -14.4 & -.37 & .000 \\
\hline RE & 189 & 366.6 & & & \\
\hline DSA & 170 & 138.13 & -1.25 & -.032 & .000 \\
\hline RE & 189 & 217.66 & & & \\
\hline
\end{tabular}

Nota. Siglas y abreviaturas: S/ NEAE - Sin/ Necesidades Específicas de Apoyo Educativo; DI/DD - Discapacidad Intelectual y otras Discapacidades del Desarrollo; DSA - Dificultades Significativas de Aprendizaje; RE - Retraso Escolar; ACI/PDC - Adaptación Curricular Individualizada/ Programa de Diversificación Curricular; Ref.Ed. - Refuerzo Educativo; AF - Agrupamiento Flexible.

Se observan diferencias significativas en todos los grupos contrastados $(p<.000)$, evidenciando el desequilibrio en términos de autodeterminación y sus componentes (autonomía, autorregulación, empoderamiento y autoconocimiento) que va en detrimento del alumnado más 
vulnerable: el alumnado con discapacidad y el alumnado con Dificultades Significativas de Aprendizaje (DSA).

Existen notable diferencias en el contraste entre alumnado sin NEAE y el alumnado con Discapacidad Intelectual y del Desarrollo (DI/DD) con una magnitud de efecto alta en todos ellos $(d>-.55)$, a excepción del autoconocimiento $(Z=-14.45 ; p<.000 ; d=-.37)$. Este dato nos indica que el alumnado con DI/DD percibe un menor control de su vida, autonomía, capacidad para autorregularse, empoderamiento y autconocimiento respecto al alumnado sin NEAE. Resultados similares se observan en el contraste entre alumnado sin NEAE y con DSA en autodeterminación (tabla 5) y en autonomía ( $Z=-15.56 ; p<.000 ; d=-.4)$, observándose valores con un efecto pequeño en el resto de componentes $(d<-.3)$. Las diferencias entre alumnado con discapacidad y con Retraso Escolar (RE) también son notables, siendo éste último quien puntúa más alto en autonomía $(Z=-13.36 ; \quad p<.000 ; \quad d=-.34)$, autorregulación $(Z=-12.3 ; \quad p<.000 ; \quad d=-.32)$ y empoderamiento $(Z=-11.59 ; p<.000 ; d=-.3)$, habiendo también diferencias significativas en el autoconocimiento (superior para el alumnado con RE; $Z=-7.99 ; p<.000 ; d=-.21$ ).

También hay diferencias estadísticamente significativas entre alumnado con Dificultades Significativas de Aprendizaje y con Refuerzo Educativo en todas las variables contrastadas, pero la magnitud del efecto es inferior a $d=.1$.

Estos resultados deben ser contextualizados en un ambiente de apoyos que, en función de sus características y configuración, pueden ser una ayuda o una barrera hacia la consecución de una plena autodeterminación del alumnado. Cabe recordar los datos presentados en la tabla 1, que exponía que las medidas educativas más empleadas en los centros educativos son:

- El refuerzo educativo, sobre todo en alumnado con Dificultades Significativas de Aprendizaje (DSA) y Refuerzo Educativo (RE).

- Las Adaptaciones Curriculares Individualizadas (ACl) o los Programas de Diversificación Curricular (PDC), mayoritariamente en alumnado con Discapacidad Intelectual y Del Desarrollo (DI/DD).

- El agrupamiento flexible, sobre todo en alumnado con Retraso Escolar (RE).

- La combinación de agrupamiento flexible (Agr.FI.) y refuerzo educativo (Ref.Ed.), utilizadas de forma mayoritaria con alumnado con Discapacidad Intelectual y Del Desarrollo.

Además, existe un $14.2 \%$ de los casos en los que no se toman medidas de ningún tipo con este alumnado, destacando 27 alumnos/as con discapacidad que no reciben apoyo de ningún tipo.

Para contrastar la influencia de los apoyos se analiza únicamente el grupo de alumnado con NEAE. La prueba $\mathrm{H}$ de Kruskal-Wallis muestra diferencias significativas en autodeterminación $\left(\chi^{2}\right.$ $=30.287 ; p=.000)$, autonomía $\left(\chi^{2}=30.939 ; p=.000\right)$, autorregulación $\left(\chi^{2}=27.581 ; p=.000\right)$, empoderamiento $\left(\chi^{2}=20.385 ; p=.000\right)$ y autoconocimiento $\left(\chi^{2}=13.371 ; p=.000\right)$. Utilizamos la $U$ de Mann-Whitney para comprobar en qué grupos residen diferencias en los niveles de autodeterminación (tabla 6) y en sus componentes (tabla 7). 
TABLA 6. Comparación de los niveles de autodeterminación en función del tipo de medida

\begin{tabular}{lccccc}
\hline & \multicolumn{5}{c}{ Autodeterminación } \\
\cline { 2 - 6 } & $N$ & Rango promedio & $Z$ & $d$ & $P$ \\
\hline ACl/PDC & 114 & 159.59 & -2.60 & -.11 & .009 \\
Ref. Ed. & 246 & 190.19 & -2.363 & .10 & .018 \\
\hline ACl/PDC & 114 & 87.81 & & & \\
Agr. Fl. & 76 & 107.04 & -3.324 & -.14 & .001 \\
\hline ACl/PDC & 114 & 87.62 & -4.774 & -.20 & .000 \\
Ref. + Agr. & 45 & 60.69 & & & \\
\hline Ref- Ed. & 246 & 156.07 & -4.743 & -.20 & .000 \\
Ref. + Agr. & 47 & 90.94 & & & \\
\hline Agr. Flex. & 76 & 72.63 & & & \\
Ref. + Agr. & 45 & 41.36 &
\end{tabular}

Nota: se omiten los resultados de la prueba $U$ en el contraste entre el grupo Ref. Ed. y el grupo Agr. Flex. pues no hay diferencias estadísticamente significativas entre esos grupos. Siglas y abreviaturas: ACI/PDC - Adaptación Curricular Individualizada/ Programa de Diversificación Curricular; Ref. Ed. - Refuerzo Educativo; Agr. Fl. - Agrupamiento Flexible.

En términos globales, se observan diferencias significativas $(p<.05)$ en el nivel de autodeterminación del alumnado con NEAE en función del tipo de apoyo recibido. La magnitud del efecto es, en todos los casos, moderada $(d>.1<.3)$. Existe un nivel superior de autodeterminación en el alumnado que recibe refuerzo educativo frente al resto de medidas. El alumnado apoyado con medidas más intensivas (ACI/PDC y combinación de agrupamiento flexible y refuerzo educativo) presenta un nivel inferior de autodeterminación.

TABLA 7. Comparación de las secciones de la autodeterminación en función del tipo de medida ${ }^{\mathrm{a}}$

\begin{tabular}{|c|c|c|c|c|c|c|c|c|c|c|c|}
\hline & $N$ & $\begin{array}{c}\text { Rango } \\
\text { promedio }\end{array}$ & $z$ & $d$ & $P$ & & $N$ & $\begin{array}{c}\text { Rango } \\
\text { promedio }\end{array}$ & $Z$ & $d$ & $P$ \\
\hline \multicolumn{6}{|c|}{ Autonomía } & \multicolumn{6}{|c|}{ Autorregulación } \\
\hline $\begin{array}{l}\text { ACl/PDC } \\
\text { Ref. Ed. }\end{array}$ & $\begin{array}{l}114 \\
246\end{array}$ & $\begin{array}{l}154.77 \\
192.42\end{array}$ & -3.19 & -.13 & .001 & $\begin{array}{l}\text { ACI/PDC } \\
\text { Ref. + Agr. }\end{array}$ & $\begin{array}{r}114 \\
45\end{array}$ & $\begin{array}{l}90.77 \\
57.31\end{array}$ & -4.16 & -.18 & .000 \\
\hline $\begin{array}{l}\text { ACl/PDC } \\
\text { Agr. Fl. }\end{array}$ & $\begin{array}{r}114 \\
76\end{array}$ & $\begin{array}{r}88.15 \\
106.53\end{array}$ & -2.26 & -.10 & .024 & $\begin{array}{l}\text { Ref. Ed. } \\
\text { Ref. + Agr. }\end{array}$ & $\begin{array}{r}246 \\
47\end{array}$ & $\begin{array}{r}157.74 \\
90.78\end{array}$ & -4.99 & -.21 & .000 \\
\hline $\begin{array}{l}\text { ACl/PDC } \\
\text { Ref. + Agr. }\end{array}$ & $\begin{array}{r}114 \\
45\end{array}$ & $\begin{array}{r}87.71 \\
64.72\end{array}$ & -2.85 & -.12 & .004 & $\begin{array}{l}\text { Agr. Flex. } \\
\text { Ref. + Agr. }\end{array}$ & $\begin{array}{l}76 \\
45\end{array}$ & $\begin{array}{l}73.29 \\
57.31\end{array}$ & -4.49 & -.19 & .000 \\
\hline $\begin{array}{l}\text { Ref. Ed. } \\
\text { Ref. + Agr. }\end{array}$ & $\begin{array}{r}246 \\
47 \\
\end{array}$ & $\begin{array}{r}157.43 \\
92.38 \\
\end{array}$ & -4.83 & -.20 & .000 & & & & & & \\
\hline $\begin{array}{l}\text { Agr. Flex. } \\
\text { Ref. + Agr. }\end{array}$ & $\begin{array}{l}76 \\
45\end{array}$ & $\begin{array}{l}72.49 \\
45.03\end{array}$ & -4.16 & -.18 & .000 & & & & & & \\
\hline \multicolumn{6}{|c|}{ Emponderamiento } & \multicolumn{6}{|c|}{ Autoconcepto } \\
\hline $\begin{array}{l}\text { ACl/PDC } \\
\text { Ref. + Agr. }\end{array}$ & $\begin{array}{r}114 \\
45\end{array}$ & $\begin{array}{l}88.14 \\
63.67\end{array}$ & -3.03 & -.13 & .002 & $\begin{array}{l}\text { ACl/PDC } \\
\text { Ref. Ed. }\end{array}$ & $\begin{array}{l}114 \\
246\end{array}$ & $\begin{array}{l}154.25 \\
192.66\end{array}$ & -3.26 & -.14 & .001 \\
\hline $\begin{array}{l}\text { Ref. Ed. } \\
\text { Ref. + Agr. }\end{array}$ & $\begin{array}{r}246 \\
47 \\
\end{array}$ & $\begin{array}{l}155.66 \\
101.65 \\
\end{array}$ & -4.01 & -.17 & .000 & $\begin{array}{l}\text { ACl/PDC } \\
\text { Agr. Fl. }\end{array}$ & $\begin{array}{r}114 \\
76\end{array}$ & $\begin{array}{r}86.32 \\
109.28 \\
\end{array}$ & -2.83 & -.12 & .005 \\
\hline $\begin{array}{l}\text { Agr. Flex. } \\
\text { Ref. + Agr. }\end{array}$ & $\begin{array}{l}76 \\
45\end{array}$ & $\begin{array}{l}72.57 \\
63.67\end{array}$ & -4.19 & -.18 & .000 & & & & & & \\
\hline
\end{tabular}

Nota:

a. se omiten los resultados de la prueba $U$ en el contraste entre el grupo Ref. Ed. y el grupo Agr. Flex. pues no hay diferencias estadísticamente significativas entre esos grupos.

b. La tabla únicamente muestra resultados estadísticamente significativos. Se omiten los datos no significativos.

c. Siglas y abreviaturas: ACI/PDC - Adaptación Curricular Individualizada/ Programa de Diversificación Curricular; Ref. Ed. Refuerzo Educativo; Agr. Fl. - Agrupamiento Flexible. 
En el contraste entre las características de la autodeterminación, el alumnado que recibe refuerzo educativo experimenta superiores niveles de autonomía, autorregulación y empoderamiento, frente al que recibe $A C I / P D C$ y al que tiene medidas combinadas de agrupamiento y refuerzo. El alumnado con ACI/PDC experimenta niveles superiores de autonomía, autorregulación y empoderamiento que el alumnado con medidas combinadas de refuerzo y agrupamiento. En términos de autoconocimiento las diferencias solo se evidencian entre alumnado con $\mathrm{ACI} / \mathrm{PDC}$, con refuerzo educativo y con agrupamiento flexible. La magnitud del efecto en todos los contrastes significativos es moderada $(d>.1<.3)$.

\section{Conclusiones}

De acuerdo con lo observado en los resultados, a nivel general, el alumnado gallego se siente autodeterminado en tanto que el promedio de las puntuaciones se sitúa en torno al $75 \%$, siendo superior en términos de autoconocimiento y empoderamiento (P.E. $>75 \%$ ) lo que indica que su aprendizaje y expresión, tanto por el trabajo efectuado en el contexto familiar como en el escolar, está teniendo resultados satisfactorios. Estos resultados concuerdan con los obtenidos en estudios previos como los de Gómez-Vela et al. (2012), o Wehmeyer (1995).

Sin embargo, el alumnado con NEAE presenta resultados significativamente inferiores a sus iguales sin NEAE en todas las áreas estudiadas, sobre todo el alumnado con Discapacidad Intelectual y Del Desarrollo y con Dificultades Significativas de Aprendizaje, Io cual concuerda con estudios previos que revelan el carácter predictor de tipo de discapacidad sobre los entornos educativos y de hogar en que se desenvuelve el joven con alteraciones del desarrollo intelectual, al limitar o aumentar sus oportunidades para tomar decisiones y ser más autodeterminado, así como su relación con la necesidad de apoyo, las oportunidades que el entorno le ofrezca para elegir y la existencia de un adecuado entorno de apoyos (Chambers, Wehmeyer, Saito, Lida, Lee y Singh, 2007; Chou, Wehmeyer, Palmer y Lee, 2016; Peralta y Arellano, 2014; Shogren et al., 2013).

En consonancia con lo anterior, es destacable la influencia de las medidas específicas de apoyo educativo, sobre todo en relación a su intensidad. Las medidas de carácter más intenso, como pueden ser la Adaptación Curricular Individualizada o los Programas de Diversificación Curricular en los centros ordinarios dan lugar a niveles más bajos de autodeterminación en todos sus componentes. Del mismo modo, las medidas combinadas de agrupamiento flexible y refuerzo educativo repercuten negativamente sobre el nivel de autodeterminación y sobre la autonomía, autorregulación y empoderamiento, incluso en mayor medida que la ACl o los PDC. Por el contrario, los niveles son superiores en el caso de alumnado que recibe refuerzo educativo frente al que tiene $\mathrm{ACI}$ o PDC, y a aquel con medidas combinadas de refuerzo y agrupamiento. Estos resultados pueden dar lugar a una doble interpretación:

Por un lado, parecen indicar que los contextos educativos inclusivos, en los que se promueve la utilización de medidas específicas de apoyo educativo para la permanencia de todo el alumnado en los centros no están resultando el lugar más adecuado para la promoción de la autodeterminación, tal y como ya ha sido evidenciado por estudios como el de Chambers et al. (2007), el de Shogren et al. (2007) o el de Vega, Gómez, Fernández y Badía (2013).

Por el otro lado, estas medidas suponen, en la mayoría de los casos, una desigualdad que se materializa en un acceso diferente al aprendizaje y una desigual participación del alumnado en las 
aulas. Se trata de medidas paliativas o de compensación, que alejan al alumnado con NEAE de las experiencias de aprendizaje en el aula y suponen un currículum en paralelo que limita la interacción del alumno con sus iguales en un mismo entorno de aprendizaje (Arnáiz, 2009; Domínguez et al., 2016; González Gil, 2011; Muntaner, 2010). Frente a este tipo de medidas, que poco ayudan al desarrollo integral del alumnado, a su aprendizaje autónomo y autorregulado y que minan su capacidad de creerse capaces de hacer y sentirse dueños de sus vidas, el profesorado tiene la labor de apoyar al alumnado en su aprendizaje, facilitando que desarrolle un currículum común con el de sus compañeros, reconocer y celebrar la diversidad potenciando las redes de apoyo entre iguales y proporcionando a todo el alumnado un entorno en el que se sienta respetado, valorado y comprendido (Luque y Luque, 2015; Vega et al., 2013).

También es necesario mencionar ciertas limitaciones. En primer lugar, pese a que se trata de una muestra amplia, presenta limitaciones geográficas dado que se concentra en la Comunidad Autónoma de Galicia; así mismo, destaca la heterogeneidad de la muestra, tanto para el alumnado con NEAE como sin ellas; finalmente, la variedad de medidas de apoyo aplicadas en los centros educativos que nos han llevado a unificar algunas de ellas, como por ejemplo, equiparar las $\mathrm{ACl}$ en el $1^{\circ}$ ciclo de la ESO con los PDC, utilizados en $2^{\circ}$ ciclo. Destacar también que, en la actualidad, la LOMCE (2013) ha suprimido los PDC por "Programas de Mejora del Aprendizaje y Rendimiento". Aun así, consideramos que estos resultados ofrecen información relevante para avanzar en el conocimiento sobre el nivel de autodeterminación del alumnado y, a partir de ahí, poder desarrollar estrategias para su mantenimiento y mejora.

Finalizamos este artículo ofreciendo algunas ideas que surgen de los datos obtenidos y que pueden ser de utilidad para encauzar las prácticas educativas hacia la enseñanza de habilidades relacionadas con la autodeterminación. El primer aspecto en que se debe incidir es en la toma de conciencia por parte de la comunidad educativa en general sobre la importancia de dotar al alumnado de las habilidades y competencias que les permitan alcanzar niveles superiores de autodeterminación. Para ello, se les debe reconocer y demostrar que los logros que alcancen no solo tendrán un efecto inmediato, sino que podrían repercutir sobre la obtención de logros futuros o resultados positivos deseados y, en definitiva, sobre la mejora de su calidad de vida (Erickson et al., 2015; Shogren y Shaw, 2016; Shogren et al., 2015; Wehmeyer et al., 2011).

La existencia de un entorno facilitador y de una red de apoyos adecuada, sobre todo, familiar y escolar, capacitará al adolescente para ser el agente causal de su propia vida, mientras que su incapacitación tendrá un efecto perverso sobre el desarrollo de su capacidad para tomar decisiones, controlar sobre su vida y actuar según sus preferencias, sobre todo cuando esta habilidad está en pleno desarrollo (Shogren et al., 2013; Wehmeyer et al., 2011). No obstante, es sabida la existencia de condicionantes como la falta de información y formación, de tiempo y recursos que pueden tergiversar los deseos del profesorado de potenciar el desarrollo integral, sobre todo en la etapa secundaria, donde el énfasis está puesto en los contenidos curriculares frente a la enseñanza en valores, actitudes y responsabilidades (Arnáiz, 2009; Pallisera et al., 2014; Peralta y Arellano, 2014).

Así pues, consideramos esencial orientar las prácticas educativas hacia la enseñanza de las habilidades relacionadas con la autodeterminación como contenido transversal a las materias del currículo, mediante acciones, experiencias y oportunidades adaptadas a las características particulares, necesidades y potencial de desarrollo de cada estudiante. El profesorado y el equipo de orientación deben coordinarse para:

- Evaluar el desarrollo de cada alumno/a en su contexto: toda intervención debe partir de una evaluación activa y continua, centrada en los aspectos ecológicos de la NEAE definiéndola dentro del contexto de las potencialidades y limitaciones funcionales y del contexto social. Solo así podremos adaptarnos a las cambiantes circunstancias del ambiente y proporcionar al alumnado las estrategias adicionales para afrontar nuevas 
situaciones. No es solo de evaluar los aprendizajes académicos, sino valorar su historial de aprendizaje, el contexto socio-económico y familiar en que se desarrolla, su estilo de aprendizaje, y las áreas en las que presenta mayores potencialidades y dificultades.

- Comprender la NEAE de forma individual y en el contexto de su grupo de referencia: La atención a la diversidad juega un papel clave como herramienta de actuación individualizada para estudiar las necesidades de cada alumno/a como ser único, diferente, pero con características comunes que le permiten ajustarse a los requerimientos comunes del aula y, con los adecuados apoyos, desarrollar su potencial en un contexto de inclusión total.

- Diseñar una propuesta curricular adaptada a sus necesidades: todo el alumnado tiene necesidades comunes e individuales que deben ser satisfechas, pero para el alumnado con NEAE son más evidentes. Las escuelas deben enseñar no solo conocimientos conceptuales (saber: hechos, datos, principios), sino también procedimentales (saber hacer: estrategias, técnicas) y actitudinales (saber ser: valores, creencias). Las medidas de apoyo están diseñadas fundamentalmente para el desarrollo del saber y, en menor medida, del saber hacer, siendo relegado a un segundo plano el saber ser. Entonces ¿cómo es posible unificar en una sola propuesta curricular los tres saberes? El aprendizaje procedimental $\mathrm{y}$, sobre todo, actitudinal en la adolescencia tiene como base la identificación con su grupo de iguales. En interacción con los compañeros, tendrá oportunidad de poner a prueba sus conocimientos (saber hacer) y mostrar su punto de vista. Saber hacer y saber ser son elementos clave en la configuración de la identidad personal, algo que sólo será posible si el alumno se siente parte de su aula. Por ello, las medidas de apoyo deben diseñarse fomentando la participación activa del alumnado y la cooperación educativa.

Por otro lado, señalar la importancia de que las intervenciones se contemplen como una tríada entre investigación, intervención y formación. Todos los agentes implicados (investigadores/as, expertos/as, agentes educativos, familias y, por supuesto, adolescentes) deben aunar sus esfuerzos para lograr convertir a la adolescencia en protagonista, de su vida. La literatura revela la influencia de las creencias y percepciones acerca de las capacidades del alumnado como facilitadoras o barreras al desarrollo de sus habilidades para ser autodeterminado (Gómez-Vela et al., 2012; Shogren et al., 2013).

En definitiva, conseguir la implicación activa del alumnado en su aprendizaje requiere que el docente actúe como facilitador de las condiciones que permitan un aprendizaje autorregulado de sus alumnos y los capacite para aumentar y mejorar sus estrategias de resolución de problemas y de afrontamiento de situaciones aumentando, de esta forma, su autoconocimiento y su empoderamiento. Este sistema de apoyo del docente en el aula podría propiciar el temprano desarrollo del adolescente y afianzar la adquisición de las habilidades relacionadas con la autodeterminación, al tiempo que ejercer un importante rol en el logro de otras metas y la obtención de resultados deseados positivos en su transición a la vida adulta, al permitirle experimentar una calidad de vida acorde con sus valores, preferencias, fortalezas y necesidades.

\section{Referencias bibliográficas}

Hernández Pina, F. (1995). Bases metodológicas de la investigación educativa. I. Fundamentos. Barcelona: PPU. 
Manzano, V. y Braña, T. (2003). Análisis de datos y técnicas de muestreo. En J.P. Lévi y J. Varela (Dirs.), Análisis multivariable para las ciencias sociales (pp. 91-144). Madrid: Pearson.

Muntaner, J.L. (2010). De la integración a la inclusión: un nuevo modelo educativo. En P. Arnáiz, M.D. Hurtado y F.J. Soto (Coords.). 25 años de integración escolar en España. Tecnología e inclusión en el ámbito educativo, laboral y comunitario. Murcia: Universidad de Murcia.

Schalock, R.L., Buntix, R., Borthwick-Duffy, S., Bradley, V., Buntix, W., Coulter, D. Yeager, M. (2010). Intellectual disability: definition, classification, and systems supports $\left(11^{\mathrm{a}} \mathrm{ed}.\right)$. Washington, EE.UU.: AAIDD.

Wehmeyer, M.L. (1995). The arc's self-determination scale: procedural guidelines. Texas, EE.UU.: The Arc National of United States.

Ley Orgánica 2/2006, de 3 de mayo, de educación (LOE). BOE, núm. 106, de 4 de mayo, pp. 17158-17207.

Ley Orgánica, 8/2013, de 9 de diciembre, de mejora de la calidad educativa (LOMCE). BOE, de 10 de diciembre, pp. 97858-97921.

Wehmeyer, M.L. (2003). A functional theory of self-determination: model overview. En M.L. Wehmeyer, B. Abery, D.E. Mithaug y R. Stancliffe (Eds.), Theory in Self-Determination: foudations for Educational Practice (pp. 182-201). Springfield, IL: Charles C.Thomas.

Wehmeyer, M.L., Field, S. y Thoma, C.A. (2012). Self-determination and adolescent transition education. En M.L. Wehmeyer y K.W. Webb (Coords.), Handbook of adolescent transition education for youth with disabilities (pp. 171-190). Londres, Reino Unido: Routledge.

Wehmeyer, M.L. y Kelchner, K. (1995). The Arc's self-determination scale. Texas, EE.UU.: The Arc National of United States.

\section{Fuentes electrónicas}

Arnáiz, P. (2009). Análisis de las medidas de atención a la diversidad en la Educación Secundaria Obligatoria. Revista de Educación, 349, 203-224. Recuperado de http://www.revistaeducacion.mec.es/re349/re349.pdf (16 febrero 2016).

Beadle-Brown, J., Leight, J., Whelton, B., Richardson, L., Beecham, J., Baumker, T. y Bradshaw, J. (2015). Quality of life and quality of support for people with severe intellectual disability and complex needs. Journal of Applied Research in Intellectual Disabilities, 1, 1-13. doi: 10.1111/jar.12200. Recuperado http://onlinelibrary.wiley.com/doi/10.1111/jar.12200/abstract (12 abril 2016).

Chambers, C.R., Wehmeyer, M.L., Saito, Y., Lida, K.M., Lee, Y. y Singh, V. (2007). Selfdetermination: What do we know? Where do we go?. Exceptionally, 15(1), 3-15. doi: 10.1080/09362830709336922. Recuperado de http://www.tandfonline.com/doi/abs/10.1080/09362830709336922 (4 de abril 2016).

Domínguez, J., López, A. y Vázquez, E. (2016). La atención a la diversidad en la educación secundaria obligatoria: análisis desde la inspección educativa. Aula Abierta. doi: 10.1016/j.aula.2016.03.002. Recuperado de http://www.sciencedirect.com/science/article/pii/S0210277316300026 (11 junio 2017).

Erickson, A.S.G., Noonan, P.M., Zheng, C. y Brussow, J.A. (2015). The relationship between selfdetermination and academic achievement for adolescents with intellectual disabilities. Research in Developmental Disabilities, 36, 45-54. doi: 10.1016/j.ridd.2014.09.008. 
Recuperado de http://www.sciencedirect.com/science/article/pii/S0891422214003989 febrero 2016).

Gómez-Vela, M., Verdugo, M.A., González-Gil, F., Badía, M. y Wehmeyer, M.L. (2012). Assessment of self-determination of Spanish students with intellectual and other educational needs. Journal of Applied Research in Intellectual Disabilities, 23(5), 505-506. Recuperado de www.ncbi.nlm.nih.gov/pmc/articles/PMC4104407/ (1 abril 2016).

González Gil, F. (2011). Inclusión y atención al alumnado con necesidades educativas especiales en España. CEE Participación Educativa, 18, 60-78. Recuperado de http://www.mecd.gob.es/revista-cee/pdf/n18-gonzalez-gil.pdf (16 febrero 2016).

Instituto Galego de Estadística. (2015). Ensinanza non universitaria. Alumnado matriculado en centros segundo nivel de ensinanza do centro [Archivo de datos y libro de códigos]. Recuperado de http://www.ige.eu/igebdt/selector.jsp?COD=463\&paxina=001\&c=0203002 (29 marzo 2016).

Luque, D.J. y Luque, M.J. (2015). Alumnado con necesidades específicas de apoyo educativo: Aspectos psicopedagógicos en un marco inclusivo. Perspectiva Educacional, 54(2), 59-73. Recuperado http://www.perspectivaeducacional.cl/index.php/peducacional/article/viewFile/333/164 (12 febrero 2016).

Marfull-Jensen, M., Flanagan, T.D. y Ossa, C. (2013). Desarrollo de habilidades para la vida: promoción de la autodeterminación en jóvenes con discapacidad. Revista Iberoamericana de Educación, 63, 51-62. Recuperado de http://www.rieoei.org/rie63a03.pdf (16 marzo 2016).

Pallisera, M., Fullana, J., Vilà, M., Jiménez, P., Castro, M., Puyalto, C... Martín, R. (2014). Análisis de los apoyos que recibe los jóvenes con discapacidad intelectual en su transición a la vida adulta en España: una investigación a partir de experiencias de profesionales y personas con discapacidad. Revista Española de Discapacidad, 2(2), 27-43. doi: 10.5569/23405104.02.02.02. Recuperado de http://www.cedd.net/redis/index.php/redis/article/view/104 (12 marzo 2016).

Peralta, F. y Arellano, A. (2014). La autodeterminación de las personas con discapacidad intelectual: situación actual en España. Revista CES Psicología, 7(2), 59-77. Recuperado de http://revistas.ces.edu.co/index.php/psicologia/article/view/2891/2205 (1 marzo 2016).

Rodríguez, A.M. (2013). Modelos de atención a la diversidad en Educación Secundaria Obligatoria: Análisis comparativo de los planes de atención a la diversidad de las comunidades autónomas de Andalucía y de la región de Murcia. Revista Nacional e Internacional de Educación Inclusiva, 6(3), 41-64. Recuperado de http://riberdis.cedd.net/handle/11181/4235?locale-attribute=pt (16 de febrero 2016).

Shogren, K.A. y Shaw, L.A. (2016). The role of autonomy, self-realization, and psychological empowerment in predicting outcomes for youth with disabilities. Remedial and Special Education, 37(1), 55-62. doi: 10.1177/0741932515585003. Recuperado de http://rse.sagepub.com/content/early/2015/06/08/0741932515585003.abstract (12 marzo 2016).

Shogren, K.A., Wehmeyer, M.L., Palmer, S.B. y Paek, Y. (2013). Exploring personal and school environment characteristics that predict self-determination. Exceptionality: a Special Education Journal, 21(3), 147-157. doi: 10.1080/09362835.2013.802231. Recuperado de http://www.tandfonline.com/doi/abs/10.1080/09362835.2013.802231\#.VyDjjISLTcs (27 marzo 2016).

Shogren, K.A., Wehmeyer, M.L., Palmer, S.B., Rifenbark, G. y Little, T. (2015). Relationships between self-determination and postschool outcomes for youth with disabilities. Journal of 
Special Education, 48(4), 256-267. doi: 10.1177/0022466913489733. Recuperado de http://sed.sagepub.com/content/early/2013/06/24/0022466913489733 (1 abril 2016).

Shogren, K.A., Wehmeyer, M.L., Palmer, S.B., Soukup, J.H., Little, T.D., Garner, N. y Lawrence, M. (2007). Examining individual and ecological predictors of the self-determination of students with disabilities. Exceptional Children, 73(4), 488-509. Recuperado de eirc.ed.gov/?id=EJ817517 (4 abril 2016).

Vega, C., Gómez, M., Fernández, R. y Badía, M. (2013). El papel del contexto educativo en la autodeterminación. Análisis de su influencia en el proceso de transición a la vida adulta de alumnos con discapacidad intelectual. Revista Iberoamericana de Educación, 63, 19-33. Recuperado http://riberdis.cedd.net/bitstream/handle/11181/4359/El_papel_del_contexto_educativo.pdf?se quence $=1 \&$ rd=0031371311959578 (4 marzo 2016).

Verdugo, M. A., Vicente, E., Fernández, R. Gómez-Vela, M., y Guillén, V. (2015a). A psychometric evaluation of the ARC-INICO Self-Determination Scale for adolescents with intellectual disabilities. International Journal of Clinical and Health Psychology, 15(2), 149-159 Recuperado de http://www.elsevier.es/es-revista-international-journal-of-clinical-and-355resumen-evaluacion-psicometrica-escala-arc-inico-autodeterminacion-90421842 (11 junio 2017).

Verdugo, M. A., Vicente, E., Gómez-Vela, M., Fernández, R., Wehmeyer, M.L. y Badia, M., González-Gil, F. y Calvo, M. I. (2015b). Escala ARC-INICO de Evaluación de la Autodeterminación. Manual de aplicación y corrección. Salamanca: Publicaciones del INICO Recuperado de http://sid.usal.es/idocs/F8/FDO26898/herramientas_autodeterminacion.pdf (11 junio 2017).

Verdugo, M.A., Gómez-Vela, M., Fernández, R., Vicente, E., Wehmeyer, M.L., Badía, M. Guillén, V. (2014). ¿Cómo evaluar la autodeterminación? Escala arc-inico de evaluación de la autodeterminación. Siglo Cero, 44(4), 21-39. Recuperado de http://sid.usal.es/20316/8-2-6 (23 noviembre 2015).

Vicente, E., Verdugo, M.A., Gómez, M., Fernández, R. y Guillén, V. (2015). Propiedades psicométricas de la escala ARC-INICO para evaluar la autodeterminación. Revista Española de Orientación y Psicopedagogía, 26(1), 8-24. Recuperado de http://revistas.uned.es/index.php/reop/article/view/14339/12829 (20 enero 2016).

Wehmeyer, M.L. (1999). A functional model of self-determination: describing development and implementing instruction. Focus on Autism and Other Developmental Disabilities, 14(1), 53-61. doi: $\quad 10.1177 / 108835769901400107 . \quad$ Recuperado de http://foa.sagepub.com/content/14/1/53.abstract (25 noviembre 2015).

Wehmeyer, M.L. y Abery, B. (2013). Self-determination and choice. Intellectual and Developmental Disabilities, 51(5), 399-411. doi: 10.1352/1934-9556-51.5.399. Recuperado de http://www.ncbi.nlm.nih.gov/pubmed/24303826 (4 febrero 2016).

Wehmeyer, M.L., Abery, B.H., Zhang, D., Ward, K., Willis, D., Hossain Amin, W., Walker, H. (2011). Personal self-determination and moderating variables that impact efforts to promote self-determination. Exceptionally, 19(1), 19-30. Recuperado de www.aucd.org/docs/ScalingUpPaper2Final.pdf (23 noviembre 2015).

Fecha de entrada: 2 Junio de 2016

Fecha de revisión: 16 Junio de 2017

Fecha de aceptación: 1 Marzo de 2018 